\title{
The Martin entrance boundary of the Galton-Watson process
}

\author{
Gerold Alsmeyer ${ }^{\mathrm{a}, *}$, Uwe Rösler ${ }^{\mathrm{b}}$ \\ a Institut für Mathematische Statistik, Fachbereich Mathematik, Westfälische Wilhelms-Universität Münster, \\ Einsteinstraße 62, 48149 Münster, Germany \\ b Mathematisches Seminar, Christian-Albrechts-Universität Kiel, Ludewig-Meyn-Straße 4, 24098 Kiel, Germany
}

Received 15 July 2004; accepted 17 May 2005

Available online 9 December 2005

\begin{abstract}
The paper provides a complete description of the Martin entrance boundary and its minimal elements for a Galton-Watson process $\left(Z_{n}\right)_{n \geqslant 0}$. Since this is easily done and known for critical processes, we deal with the noncritical case which in turn can be reduced to the subcritical one. The Martin entrance boundary consists of all quasi-invariant Radon measures. The minimal Martin entrance boundary is isomorphic to $[0,1)$ as a torus. Every element of the minimal Martin entrance boundary is uniquely identified through its generating function. These minimal quasi-invariant measures are the extremals in the simplex of quasi-invariant Radon measures. We provide explicitly the Martin topology in the set of potentials. All this is done via the Martin kernel approach and under no additional assumption on $\left(Z_{n}\right)_{n \geqslant 0}$. In particular, we do not require the $(L \log L)$-condition $\mathbb{E} Z_{1} \log Z_{1}<\infty$.

(C) 2005 Elsevier SAS. All rights reserved.
\end{abstract}

\section{Résumé}

Cet article fournit une description complète de la frontière d'entrée de Martin et de ses éléments minimaux pour un processus de Galton-Watson $\left(Z_{n}\right)_{n \geqslant 0}$. Comme ceci est facilement fait et bien connu pour des processus critiques, nous traitons seulement le cas non-critique qui peut à son tour être réduit au cas sous-critique. La frontière d'entrée de Martin consiste en toutes les mesures de Radon quasi-invariantes, et la frontière d'entrée minimale est isomorphe à l'intervalle $[0,1)$ considéré comme un cercle. Chaque élément de la frontière d'entrée minimale est uniquement identifié par sa fonction génératrice. Ces mesures minimales et quasiinvariantes sont les éléments extrêmes du simplexe des mesures de Radon quasi-invariantes. Nous déterminons explicitement la topologie de Martin dans l'ensemble des potentiels. Notre démarche est basée sur les noyaux de Martin et n'utilise pas d'hypothèses supplémentaires sur $\left(Z_{n}\right)_{n \geqslant 0}$. En particulier, nous n'utilisons pas la condition $(L \log L) \mathbb{E} Z_{1} \log Z_{1}<\infty$.

(c) 2005 Elsevier SAS. All rights reserved.

MSC: 60J80; 60J45

Keywords: Galton-Watson process; Quasi-invariant measure; Martin entrance boundary

\footnotetext{
* Corresponding author.

E-mail address: gerolda@math.uni-muenster.de (G. Alsmeyer).
} 


\section{Introduction}

The simple Galton-Watson process (GWP) $\left(Z_{n}\right)_{n \geqslant 0}$ is a temporally homogeneous Markov chain with state space $\mathbb{N}_{0}=\{0,1,2, \ldots\}$ and recursively defined as

$$
Z_{n}=\sum_{k=1}^{Z_{n-1}} X_{n, k}, \quad n \geqslant 1
$$

where the $X_{n, k}, k, n \geqslant 1$, are i.i.d. integer-valued random variables with common distribution $p=\left(p_{j}\right)_{j} \geqslant 0 . Z_{n}$ describes the size of the $n$-th generation of a population in which individuals all have life span 1 and reproduce independently according to $p$, called offspring distribution. $\left(Z_{n}\right)_{n \geqslant 0}$ has (one step) transition probabilities

$$
P(i, j) \stackrel{\text { def }}{=} \mathbb{P}\left(Z_{n}=j \mid Z_{n-1}=i\right)=\mathbb{P}\left(\sum_{k=1}^{i} X_{n, k}=j\right)=p_{j}^{* n}, \quad i, j \geqslant 0,
$$

where $p^{* n}=\left(p_{j}^{* n}\right)_{j} \geqslant 0$ denotes the $n$-fold convolution of $p$. The state 0 is absorbing and means extinction of the population. It is a classic result (see e.g. [3]) that the extinction probability $q \stackrel{\text { def }}{=} \mathbb{P}\left(Z_{n}=0\right.$ eventually $\left.\mid Z_{0}=1\right)$ given one ancestor can be identified as the smallest fixed point in $[0,1]$ of the generating function (g.f.) $f(s) \stackrel{\text { def }}{=} \sum_{j} \geqslant 0 p_{j} s^{j}$ of $p$, and that, unless $p_{1}=1, q<1$ holds iff the reproduction mean $m \stackrel{\text { def }}{=} \sum_{j \geqslant 1} j p_{j}$ is greater than 1 (supercritical case). Hence extinction occurs almost surely in the critical $(m=1)$ and subcritical $(m<1)$ case. Note that $\mathbb{P}\left(Z_{n}=0\right.$ eventually $\left.\mid Z_{0}=i\right)=q^{i}$ for all $i \geqslant 0$.

An invariant or stationary measure of $\left(Z_{n}\right)_{n \geqslant 0}$ is a measure $\mu=\left(\mu_{j}\right)_{j \geqslant 0}$ on $\mathbb{N}_{0}$ satisfying

$$
\sum_{i \geqslant 0} \mu_{i} P(i, j)=\mu_{j}, \quad j \geqslant 0
$$

We allow the $\mu_{j}$ to be $\infty$ and stipulate $0 \cdot \infty=\infty \cdot 0 \stackrel{\text { def }}{=} 0$ as usual. Note that the set $\mathcal{I}$ of all invariant measures of $\left(Z_{n}\right)_{n \geqslant 0}$ forms a convex cone $\left(\mu_{1}, \mu_{2} \in \mathcal{I} \Rightarrow \alpha \mu_{1}+\beta \mu_{2} \in \mathcal{I}\right.$ for all $\left.\alpha, \beta \geqslant 0\right)$. Of primary interest are naturally invariant Radon measures $\mu$ (which satisfy $\mu_{j}<\infty$ for all $j \geqslant 0$ ). On the other hand, it was shown by Harris [4] that $\mu=(1,0,0, \ldots)$ is the only nontrivial invariant Radon measure (modulo multiplicative constants). Given this negative result at the outset, a proper restatement of the problem is to ask for all quasi-invariant or quasi-stationary Radon measures of $\left(Z_{n}\right)_{n \geqslant 0}$ by which we mean any Radon measure $\eta=\left(\eta_{j}\right)_{j \geqslant 1}$ on the positive integers which instead of (1.2) satisfies

$$
\sum_{i \geqslant 1} \eta_{i} P(i, j)=\eta_{j}, \quad j \geqslant 1
$$

The set $\mathcal{Q}$ of all such measures forms again a convex cone. Since $P(0, j)=0$ for all $j \geqslant 1$ we see that every invariant Radon measure is also quasi-invariant when restricted to $\mathbb{N}$. The simple observation that each $\eta \in \mathcal{Q}$ can be uniquely identified with the stationary measure $\mu \in \mathcal{I}$, defined as

$$
\mu \stackrel{\text { def }}{=}\left(\infty, \eta_{1}, \eta_{2}, \ldots\right)
$$

shows that, conversely, quasi-stationary measures may also be viewed as those solutions to (1.2) which are almost Radon in that they possibly carry infinite mass only in one point, namely 0 . It is further readily verified that other solutions of this type do not exists unless $p_{1}=1$. This trivial case as well as $p_{0}=1$ is henceforth excluded.

The set $\mathcal{Q}$, which is our main concern here, generally contains nontrivial elements. The classic monographs by Athreya and Ney [3, Chapter II] and by Asmussen and Hering [2, Chapter III, Section 2] provide comprehensive studies of $\mathcal{Q}$ but appear to be incomplete in various aspects. Indeed, somewhat surprisingly in view of the vast literature on the simple GWP we were not able to find any reference where the problem of describing $\mathcal{Q}$ is solved in full generality. This fact in combination with the following probabilistic interpretation of the elements of $\mathcal{Q}$ in case $m \leqslant 1$, which played a key role in [1], provided a major motivation for the present work. 
Let $P^{n}(i, j)$ be the $n$-step transition function $\left(P^{0}(i, j) \stackrel{\text { def }}{=} \delta_{i j}\right)$,

$$
G(i, j) \stackrel{\text { def }}{=} \sum_{n \geqslant 0} P^{n}(i, j)
$$

the associated Green function and $\tau \stackrel{\text { def }}{=} \inf \left\{n \geqslant 0: Z_{n}=0\right\}$ the extinction time of $\left(Z_{n}\right)_{n} \geqslant 0$. Note that $f_{n}^{k}$, the $k$-th power of the $n$-fold iteration of $f$, gives the g.f. of $Z_{n}$ under $\mathbb{P}_{k} \stackrel{\text { def }}{=} \mathbb{P}\left(\cdot \mid Z_{0}=k\right)$. Now, if $\left(i_{n}\right)_{n} \geqslant 1$ is such that $i_{n} \rightarrow \infty$ and $\eta_{j} \stackrel{\text { def }}{=} \lim _{n \rightarrow \infty} G\left(i_{n}, j\right)<\infty$ exists for each $j \geqslant 1$, then $\eta=\left(\eta_{j}\right)_{j \geqslant 1}$ constitutes a quasi-invariant measure normalized by $\hat{\eta}\left(p_{0}\right)=1$, where $\hat{\eta}(s) \stackrel{\text { def }}{=} \sum_{j \geqslant 1} \eta_{j} s^{j}$ denotes the g.f. of $\eta$. Quasi-invariance is ensured by

$$
\begin{aligned}
\eta_{j} & =\lim _{n \rightarrow \infty} \sum_{k \geqslant 0} P^{k}\left(i_{n}, j\right)=\lim _{n \rightarrow \infty} \sum_{k \geqslant 1} \sum_{i \geqslant 1} P^{k-1}\left(i_{n}, i\right) P(i, j) \\
& =\sum_{i \geqslant 1}\left(\lim _{n \rightarrow \infty} \sum_{k \geqslant 1} P^{k-1}\left(i_{n}, i\right)\right) P(i, j)=\sum_{i \geqslant 1} \eta_{i} P(i, j), \quad j \geqslant 1
\end{aligned}
$$

(the interchange of limit and summation being nontrivial but justified by the general theory), while normalization follows from $\left(f_{0}(s) \stackrel{\text { def }}{=} s\right)$

$$
\sum_{k \geqslant 0} \sum_{j \geqslant 1} P^{k}(i, j) p_{0}^{j}=\sum_{k \geqslant 0}\left(f_{k+1}^{i}(0)-f_{k}^{i}(0)\right)=1, \quad i \geqslant 1 .
$$

An elementary computation shows that

$$
\lim _{n \rightarrow \infty} \mathbb{P}_{i_{n}}\left(Z_{\tau-1}=j\right)=\lim _{n \rightarrow \infty} G\left(i_{n}, j\right) p_{0}^{j}=\eta_{j} p_{0}^{j}, \quad j \geqslant 1,
$$

whence $\left(\eta_{j} p_{0}^{j}\right)_{j \geqslant 1}$ is the limit law of $Z_{\tau-1}$ under $\mathbb{P}_{i_{n}}$ for $n \rightarrow \infty$. This indicates that quasi-invariant measures, suitably normalized, are directly connected to the limiting behavior of the GWP at the eve of extinction when the number of ancestors increases to infinity in a suitable fashion. More generally, we showed in [1, Lemma 4.2] that the finite dimensional distributions of $\left(Z_{\tau-k}\right)_{k} \geqslant 0$ under $\mathbb{P}_{i_{n}}$ (put $Z_{-k} \stackrel{\text { def }}{=} Z_{0}$ for $k \geqslant 1$ ) converge weakly to the respective finite dimensional distributions of a Markov chain $\left(W_{n}\right)_{n \geqslant 0}$ with $W_{0}=0$ and $n$-step transition probabilities

$$
Q_{n}(i, j) \stackrel{\text { def }}{=}\left\{\begin{array}{ll}
0, & \text { if } i=j=0, \\
\eta_{j} \mathbb{P}_{j}(\tau=n), & \text { if } i=0, j \geqslant 1, \\
\frac{\eta_{j} P^{n}(j, i)}{\eta_{i}}, & \text { if } i, j \geqslant 1,
\end{array} \quad n \geqslant 1 .\right.
$$

Based on Chapter II of Athreya and Ney's monograph [3], which is still a standard source for potential theoretic aspects of GWP, we continue with a collection of known facts about $\mathcal{Q}$. Theorem II.1.2 in [3] tells us that a Radon measure $\eta=\left(\eta_{j}\right)_{j} \geqslant 1$ on the integers is quasi-invariant iff its g.f. $\hat{\eta}(s)$ is analytic for $|s|<q$ and satisfies

$$
\hat{\eta}(f(s))=\hat{\eta}\left(p_{0}\right)+\hat{\eta}(s) \text {. }
$$

In order to identify elements of $\mathcal{Q}$ that differ only by a multiplicative constant we must choose an appropriate normalization and will later on use $\hat{\eta}\left(p_{0}\right)=1$.

In the critical case $m=1$ the problem of determining $\mathcal{Q}$ is completely settled by the following result due to Papangelou [10]:

Theorem 1.1. If $m=1$ there is a unique (up to multiplicative constants) quasi-invariant measure $\pi=\left(\pi_{j}\right)_{j} \geqslant 1$. It has infinite mass and can be obtained as

$$
\pi_{j}=\lim _{n \rightarrow \infty} \frac{P^{n}(1, j)}{P^{n}(1, r)},
$$

where $r \stackrel{\text { def }}{=} \inf \left\{i \geqslant 1: p_{i}>0\right\}$ and $P^{n}(1, j) / P^{n}(1, r)$ is increasing in $n$. 
The same result appears in [3, Lemma I.7.2] for the case $r=1$, i.e. $p_{1}>0$. Existence and essential uniqueness of $\pi$ for general critical GWP were also proved by Seneta [11], but instead of (1.7) he obtained $\pi$ as

$$
\pi_{j}=\lim _{n \rightarrow \infty} \frac{\mathbb{P}_{1}\left(Z_{n}=j \mid \tau=n+k\right)}{f_{k}^{j}(0)-f_{k-1}^{j}(0)}
$$

which is independent of $k \in \mathbb{N}$. Earlier versions under stronger assumptions on $\left(p_{j}\right)_{j} \geqslant 0$ were given in [6] $(f(s)<\infty$ for some $s>1)$ and [8] $\left(\sum_{j \geqslant 1} j^{2} p_{j}<\infty\right.$, see also [3, Theorem II.7.2]).

In view of the previous result we are left with the noncritical case $m \neq 1$ and will next quickly argue that it suffices herefore to restrict to the subcritical case $m<1$.

Assuming first $m>1$ and $p_{0}=0$, we claim that the only quasi-invariant Radon measure is $\eta \equiv 0$. Indeed, since $P(i, j)=0$ for all $i>j \geqslant 1$ and $P(i, i)<1$ for all $i \geqslant 1$, any quasi-invariant $\eta=\left(\eta_{j}\right)_{j} \geqslant 1$ satisfies $\eta_{1}=\eta_{1} P(1,1)$ and thus $\eta_{1}=0$. Now $\eta_{j}=0$ for all $j \geqslant 1$ follows by induction.

If $m>1$ and $p_{0}>0$ then $0<q<1$ and $f^{*}(s) \stackrel{\text { def }}{=} q^{-1} f(s q)$ defines the g.f. of the subcritical distribution $p^{*}=$ $\left(q^{j-1} p_{j}\right)_{j \geqslant 0}$ with mean $m^{*}=f^{\prime}(q)$. It is known that a GWP $\left(Z_{n}^{*}\right)_{n} \geqslant 0$ with offspring distribution $p^{*}$ can be obtained from a GWP $\left(Z_{n}\right)_{n \geqslant 0}$ with offspring distribution $p$ by counting only individuals with a finite line of descent in the pertinent population, see [3, I.12]. The following known lemma is stated for completeness and provides a simple one-to-one correspondence between the quasi-invariant Radon measures of $\left(Z_{n}\right)_{n} \geqslant 0$ and those of $\left(Z_{n}^{*}\right)_{n} \geqslant 0$.

Lemma 1.2. Given the previous notation, a Radon measure $\eta=\left(\eta_{j}\right)_{j} \geqslant 1$ is quasi-invariant for $\left(Z_{n}\right)_{n \geqslant 0}$ iff $\eta^{*} \stackrel{\text { def }}{=}\left(q^{j} \eta_{j}\right)_{j \geqslant 1}$ is quasi-invariant for $\left(Z_{n}^{*}\right)_{n \geqslant 0}$.

Proof. Let $\eta$ be quasi-invariant for $\left(Z_{n}\right)_{n \geqslant 0}$ and w.l.o.g. $\hat{\eta}\left(p_{0}\right)=1$. Using characterization (1.6) of quasi-invariance, we obtain

$$
\hat{\eta}^{*}\left(f^{*}(s)\right)=\sum_{j \geqslant 1} \eta_{j} q^{j}\left(\frac{f(s q)}{q}\right)^{j}=\hat{\eta}(f(s q))=1+\hat{\eta}(s q)=1+\hat{\eta}^{*}(s)
$$

and thus the quasi-invariance of $\eta^{*}$ for $\left(Z_{n}^{*}\right)_{n \geqslant 0}$ by another appeal to (1.6).

Notice that $\left(Z_{n}^{*}\right)_{n} \geqslant 0$ is not only subcritical but also having moments of exponential order because its offspring distribution $p^{*}$ has geometrically decreasing tails. Theorem 2.2 in the following section will give a description of all quasi-invariant Radon measures for subcritical GWP satisfying the $(L \log L)$ moment condition. It hence applies to $\left(Z_{n}^{*}\right)_{n \geqslant 0}$ which in combination with Lemma 1.2 immediately leads to a description of all quasi-invariant Radon measures of a supercritical GWP (see Corollary 2.3).

\section{Quasi-invariant Radon measures for subcritical GWP}

After the previous review it is clear that we will now entirely focus on the subcritical case. So we are given a GWP $\left(Z_{n}\right)_{n \geqslant 0}$ with offspring distribution $p=\left(p_{j}\right)_{j \geqslant 0}$ having mean $0<m<1$ and g.f. $f$. We first assume the $(L \log L)$ moment condition

$$
\mathbb{E}_{1} Z_{1} \log Z_{1}=\sum_{j \geqslant 2} p_{j} j \log j<\infty .
$$

Defining the nonincreasing sequence

$$
\zeta_{n} \stackrel{\text { def }}{=} \frac{\mathbb{P}_{1}\left(Z_{n}>0\right)}{m^{n}}=\frac{1-f_{n}(0)}{m^{n}}
$$

for $n \geqslant 0$, we then have that

$$
\zeta \stackrel{\text { def }}{=} \lim _{n \rightarrow \infty} \zeta_{n}
$$

is positive, see [5, Theorem (2.6.1)], while $\zeta=0$ if $(L \log L)$ fails to hold. Next put

$$
Q_{n}(s) \stackrel{\text { def }}{=} \frac{f_{n}(s)-1}{\zeta m^{n}}, \quad n \geqslant 1, s \in[0,1] .
$$


As shown in [3, I.11], its derivative $Q_{n}^{\prime}(s)$ converges pointwise to a function $Q^{\prime}(s)$ for $0 \leqslant s<1$ which is positive on $(0,1)$ under $(L \log L)$. Setting

$$
Q(s) \stackrel{\text { def }}{=} \int_{1}^{s} Q^{\prime}(r) \mathrm{d} r
$$

we hence obtain $Q_{n}(s) \rightarrow Q(s)$ for $0 \leqslant s \leqslant 1$. $Q$ further satisfies $Q(0)=-1, Q(1)=0, \lim _{s \rightarrow 1} Q^{\prime}(s)=\zeta$ and is the unique solution with these properties to the functional equation

$$
Q(f(s))=m Q(s), \quad 0 \leqslant s<1,
$$

see [3, Theorem I.11.2]. The normalization of $Q(s)$ by $\zeta$ is only given here in order to unify its definition with the one below for the general situation.

Without assuming $(L \log L)$ a solution of (2.3) still exists but must be derived differently because $\zeta=0$ and $\lim _{n \rightarrow \infty} \frac{\mathrm{d}}{\mathrm{d} s}\left(\left(f_{n}(s)-1\right) / m^{n}\right) \equiv 0$ on $[0,1)$ if $\sum_{j \geqslant 2} p_{j} j \log j=\infty$. The details will be presented in Section 3 where we will show that

$$
Q(s) \stackrel{\text { def }}{=} \lim _{n \rightarrow \infty} \frac{f_{n}(s)-1}{1-f_{n}(0)}, \quad s \in[0,1),
$$

forms the unique analytic solution to (2.3) satisfying $Q(0)=-1$ and $Q(1)=0$. Hence under $(L \log L)$ the limits of $\left(f_{n}(s)-1\right) /\left(1-f_{n}(0)\right)$ and $\left(f_{n}(s)-1\right) /\left(\zeta m^{n}\right)$ are the same for all $s \in[0,1)$.

As in [3, Chapter II], we put

$$
U(s, t) \stackrel{\text { def }}{=} \sum_{n \in \mathbb{Z}}\left(\exp \left(Q(s) m^{n-t}\right)-\exp \left(Q(0) m^{n-t}\right)\right), \quad s \in[0,1), t \in \mathbb{R} .
$$

It is easily verified that this series converges and that $U(\cdot, t)$ is the g.f. of a quasi-invariant Radon measure $\eta(t)$. The quasi-invariance may be checked directly by using (1.6) and (2.3). Note also that

$$
U\left(p_{0}, t\right)=U(f(0), t)=1
$$

and

$$
U(\cdot, t)=U(\cdot, t+1)
$$

for each $t \in \mathbb{R}$. We will show that the $\eta(t)$ are the minimal elements of the convex set $\mathcal{Q}^{*} \stackrel{\text { def }}{=}\left\{\eta \in \mathcal{Q}: \hat{\eta}\left(p_{0}\right)=1\right\}$, but in order to explain this in more detail we first have to collect some facts on the general construction of the minimal Martin entrance boundary in the present context. For a more general introduction of this topic for discrete Markov chains see [7, Chapter 10].

Given any nonzero function $h: \mathbb{N} \rightarrow[0, \infty)$, put $G(i, h) \stackrel{\text { def }}{=} \sum_{j \geqslant 1} G(i, j) h(j)$ and $\eta(h) \stackrel{\text { def }}{=} \sum_{j \geqslant 1} \eta_{j} h(j)$. Let $\mathfrak{M}$ be the set $\{M(i, \cdot): i \in \mathbb{N}\}$ with

$$
M(i, j) \stackrel{\text { def }}{=} \begin{cases}\frac{G(i, j)}{G(i, h)}, & \text { if } 0<G(i, h)<\infty, \quad i, j \in \mathbb{N}, \\ 0, & \text { otherwise, }\end{cases}
$$

and $\overline{\mathfrak{M}}$ be its closure under pointwise convergence. Any sequence $\left(i_{k}\right)_{k} \geqslant 1$ of positive integers such that $i_{k} \rightarrow \infty$ and $M\left(i_{k}, \cdot\right)$ converges pointwise to some limit $\eta$ is called a Martin sequence, and $\eta$ a Martin limit relative to $h$. Every such $\eta$ is a quasi-invariant measure satisfying $\eta(h)=1$. The Martin entrance boundary (w.r.t. $h$ ) is (isomorphic to) the set $\overline{\mathfrak{M}} \backslash \mathfrak{M}$ endowed with the topology of pointwise convergence and thus equals the set of all Martin limits. By the Poisson-Martin representation theorem, each element of $\mathcal{Q}_{h} \stackrel{\text { def }}{=}\{\eta \in \mathcal{Q}: \eta(h)=1\}$ is obtained as an integral over the entrance boundary w.r.t. a probability measure which, however, does not need to be unique. An element $\eta \in \mathcal{Q}_{h}$ is called minimal or extremal, if it cannot be written as a nontrivial convex combination of two distinct elements from $\mathcal{Q}_{h}$. The minimal Martin entrance boundary is defined as the subset of minimal elements of $\mathcal{Q}_{h}$. It is necessarily a subset of $\overline{\mathfrak{M}} \backslash \mathfrak{M}$. The integral representation of a quasi-invariant (normalized) Radon measure over this minimal boundary w.r.t. a finite (probability) measure is unique. Hence there is a one-to-one correspondence between the set 
$\mathcal{Q}_{h}$ and the set of probability measures on the minimal entrance boundary. Our task is therefore to identify the minimal elements in the class of Martin limits.

The previous construction depends on the choice of $h$. With view to our goal of finding all quasi-invariant Radon measures a good choice is any $h$ such that the associated Martin entrance boundary consists exactly of all these measures (modulo positive scalars). Since every $\eta \in \mathcal{Q}$ satisfies $\hat{\eta}(f(0))<\infty$ (see before (1.6)) we fix $h(j) \stackrel{\text { def }}{=} f^{j}(0)$, $j \geqslant 1$. Then $\eta(h)=\hat{\eta}(f(0))$ and $G(\cdot, h) \equiv 1$ by (1.4) which further entails that the Martin kernel $K$ and the Green kernel $G$ are the same under this choice.

We proceed with the statement of our main result, Theorem 2.1 below, which provides an isomorphic description of the Martin entrance boundary, the Martin topology and its minimal elements for general subcritical GWP. For $x>0$ let $\alpha(x)$ and $\beta(x)$ denote the integral and fractional part of $-\log _{m} x$, respectively, where $\log _{m}$ is the $\operatorname{logarithm}$ to the base $m$. Put

$$
\tau(x) \stackrel{\text { def }}{=} \sup \left\{n \geqslant 0: 1-f_{n}(0) \geqslant 1 / x\right\}
$$

for $x \geqslant 1$ and note that $\lim _{n \rightarrow \infty}\left(\left(1-f_{n+1}(0)\right) /\left(1-f_{n}(0)\right)\right)=m$ implies $\lim \sup _{x \rightarrow \infty} \gamma(x) \in[0,1)$ for $\gamma(x) \stackrel{\text { def }}{=}$ $-\log _{m}\left(x\left(1-f_{\tau(x)}(0)\right)\right)$. Indeed, setting $\delta(x) \stackrel{\text { def }}{=} \log _{m}\left(\left(1-f_{\tau(x)+1}(0)\right) /\left(1-f_{\tau(x)}(0)\right)\right)$, we have $\lim _{x \rightarrow \infty} \delta(x)=1$ which in combination with

$$
1>x\left(1-f_{\tau(x)+1}(0)\right)=x\left(1-f_{\tau(x)}(0)\right) m^{\delta(x)}=m^{\delta(x)-\gamma(x)}
$$

gives $1-\limsup _{x \rightarrow \infty} \gamma(x)=\liminf _{x \rightarrow \infty}(\delta(x)-\gamma(x))>0$. Let $\mathbb{M} \stackrel{\text { def }}{=} \mathbb{N} \cup[0,1)$ and the function $\varphi: \mathbb{M} \rightarrow \mathbb{C}$ be defined as

$$
\varphi(x) \stackrel{\text { def }}{=} \begin{cases}\frac{x}{1+x} \mathrm{e}^{-2 \pi \mathrm{i} \log _{m}\left(x \zeta_{\tau(x)}\right)}, & \text { if } x \in \mathbb{N}, \\ \mathrm{e}^{2 \pi \mathrm{i} x}, & \text { if } x \in[0,1),\end{cases}
$$

where $\mathrm{i} \stackrel{\text { def }}{=} \sqrt{-1}$. We note that $x \zeta_{\tau(x)}$ may be replaced with $x\left(1-f_{\tau(x)}(0)\right)$ in the definition of $\varphi$ because both values differ only by an integral power of $m$. It follows that

$$
\rho(x, y) \stackrel{\text { def }}{=}|\varphi(x)-\varphi(y)|, \quad x, y \in \mathbb{M},
$$

is a metric on $\mathbb{M}$ and that $\mathbb{M}$ forms the closure of $\mathbb{N}$ w.r.t. $\rho$. $(\mathbb{M}, \rho)$ is a compact space and $[0,1)$ is endowed with the spherical topology. An integer sequence $\left(i_{n}\right)_{n} \geqslant 1$ with $i_{n} \rightarrow \infty$ converges to $t \in(0,1)$, respectively $t=0$ w.r.t. $\rho$ iff the fractional part of $-\log _{m}\left(i_{n} \zeta_{\tau\left(i_{n}\right)}\right)$ converges to $t$, as $n \rightarrow \infty$, respectively has the accumulation points 0 and/or 1 . The very last fact is not true for the metric given in [12], [2, p. 71] and [3, p. 69].

Recall that $\eta(t)$ is the quasi-invariant Radon measure with g.f. $U(\cdot, t)$ given in $(2.5)$.

Theorem 2.1. Given a subcritical GWP $\left(Z_{n}\right)_{n \geqslant 0}$, its Martin entrance boundary equals the set $\{\eta(t): t \in[0,1)\}$ and is isomorphic to $([0,1), \rho)$, the latter established by the equivalence

$$
\lim _{k \rightarrow \infty} G\left(i_{k}, \cdot\right) \rightarrow \eta(t) \text { pointwise } \Leftrightarrow \lim _{k \rightarrow \infty} \rho\left(i_{k}, t\right)=0 .
$$

All $\eta(t), t \in[0,1)$, are pairwise distinct and minimal, i.e. their collection also constitutes the minimal Martin entrance boundary. There is a bijection $v \leftrightarrow \eta$ between the set $\mathcal{Q}$ of quasi-invariant Radon measures $\eta=\left(\eta_{j}\right)_{j} \geqslant 1$ and the set of finite Borel measures $v$ on $[0,1)$, given by the integral representation

$$
\eta_{j}=\int_{[0,1)} \eta_{j}(t) v(\mathrm{~d} t), \quad j \in \mathbb{N} .
$$

A partial version of this result appears in Athreya and Ney [3, Theorem II.2.3] which asserts that under $(L \log L)$ the $\eta(t)$ are Martin limits and that any other quasi-invariant $\eta$ has an integral representation (2.8) for some not necessarily unique finite Borel measure $v$. The same result was stated earlier (without proof) by Spitzer [12] for the case $0<$ $p_{0}<p_{0}+p_{1}=1$, and later by Asmussen and Hering [2, Corollary III.2.10] without any moment condition. However, their proof, see [2, p. 70f], misses out the relevant details at exactly the point where the $(L \log L)$ condition should 
have entered. Namely, their conclusion that $\sum_{j} G\left(i_{n}, j\right) s^{j}$ converges to $U(s, t)$ if $i_{n} \rightarrow \infty$ and the fractional part of $-\log _{m} i_{n}$ converges to $t$ fails to hold in general. This follows from our analysis presented in Section 5. It is also to be emphasized that pairwise distinctness and minimality of the $v(t)$ constitute a nontrivial improvement beyond not assuming $(L \log L)$. This was needed for an essential argument in [1].

If $\left(Z_{n}\right)_{n \geqslant 0}$ satisfies the $(L \log L)$ condition one may replace $\rho$ in Theorem 2.1 with a simpler metric. Define $\varphi^{*}: \mathbb{M} \rightarrow \mathbb{C}$ as

$$
\varphi^{*}(x) \stackrel{\text { def }}{=} \begin{cases}\frac{x}{1+x} \mathrm{e}^{2 \pi \mathrm{i} \log _{m}(x \zeta)}, & \text { if } x \in \mathbb{N}, \\ \mathrm{e}^{2 \pi \mathrm{i} x}, & \text { if } x \in[0,1) .\end{cases}
$$

It then follows that

$$
\rho^{*}(x, y) \stackrel{\text { def }}{=}\left|\varphi^{*}(x)-\varphi^{*}(y)\right|, \quad x, y \in \mathbb{M},
$$

is another metric on $\mathbb{M}$. An integer sequence $\left(i_{n}\right)_{n} \geqslant 1$ with $i_{n} \rightarrow \infty$ converges to $t \in(0,1)$, respectively $t=0$ w.r.t. $\rho^{*}$ iff the fractional part of $-\log _{m}\left(\zeta i_{n}\right)=-\log \left(\zeta i_{n}\right) / \log m$ converges to $t$, as $n \rightarrow \infty$, respectively accumulates at 0 and/or 1 . Our second theorem asserts that $(\mathbb{M}, \rho)$ and $\left(\mathbb{M}, \rho^{*}\right)$ are in fact isomorphic.

Theorem 2.2. Given a subcritical GWP $\left(Z_{n}\right)_{n} \geqslant 0$ satisfying $(L \log L)$, the assertions of Theorem 2.1 remain true if the metric $\rho$ is replaced with $\rho^{*}$.

A combination of Theorem 2.2 with Lemma 1.2 immediately leads to the following result for supercritical GWP we state here for completeness.

Corollary 2.3. Given a supercritical GWP $\left(Z_{n}\right)_{n} \geqslant 0$ with associated subcritical process $\left(Z_{n}^{*}\right)_{n} \geqslant 0$ as defined in Section 1, the Martin entrance boundary $\mathfrak{M}$ of $\left(Z_{n}\right)_{n} \geqslant 0$ is isomorphic to $\left(\mathbb{M}, \rho^{*}\right)$ where $\zeta$ in the definition of $\rho^{*}$ is defined by (2.2) for $\left(Z_{n}^{*}\right)_{n \geqslant 0}$. If $\left\{\eta^{*}(t): t \in[0,1)\right\}$ denotes the minimal Martin entrance boundary of $\left(Z_{n}^{*}\right)_{n} \geqslant 0$, then there is a bijection $v \leftrightarrow \eta$ between the set $\mathcal{Q}$ of quasi-invariant Radon measures $\eta=\left(\eta_{j}\right)_{j} \geqslant 1$ of $\left(Z_{n}\right)_{n} \geqslant 0$ and the set of finite Borel measures $v$ on $[0,1)$, given by the integral representation

$$
\eta_{j}=q^{-j} \int_{[0,1)} \eta_{j}^{*}(t) v(\mathrm{~d} t), \quad j \in \mathbb{N} .
$$

We mention for historical account that Harris [4, p. 25] was the first to give an example of a quasi-invariant Radon measure in the subcritical case and that Kingman [9] first demonstrated the non-uniqueness of such measures in the supercritical case.

The proof of Theorems 2.1 and 2.2 are presented in Section 5. The existence and essential uniqueness of an analytic solution $Q(s)$ to (2.3) is shown in Section 3, while Section 4 studies a useful function in connection with the g.f. $U(\cdot, t)$ of the minimal quasi-invariant measures $\eta(t)$. In essence the results given there provide minimality and pairwise distinctness of the $\eta(t)$.

\section{The function $Q(s)$}

Given an arbitrary subcritical GWP $\left(Z_{n}\right)_{n} \geqslant 0$ with offspring mean $0<m<1$, we will now show the existence and uniqueness of an analytic function $Q(s)$ on $(-1,1)$ solving Eq. (2.3) and satisfying $Q(1)=0$ and $Q(0)=c$ for any fixed $c<0$. Define

$$
Q_{n}(s) \stackrel{\text { def }}{=} \frac{f_{n}(s)-1}{1-f_{n}(0)}, \quad n \geqslant 1, s \in[-1,1] .
$$

and note that $Q_{n}(0)=-1, Q_{n}(1)=0$. Then

$$
Q_{n}(s)=\frac{\mathbb{E}_{1}\left(s^{Z_{n}} \mathbf{1}_{\left\{Z_{n}>0\right\}}\right)-\mathbb{P}_{1}\left(Z_{n}>0\right)}{\mathbb{P}_{1}\left(Z_{n}>0\right)}=\mathbb{E}_{1}\left(s^{Z_{n}} \mid Z_{n}>0\right)-1
$$


for all $n \geqslant 1$ and $s \in[-1,1]$. By Yaglom's theorem [3, Corollary I.8.1], $\mathbb{P}_{1}\left(Z_{n} \in \cdot \mid Z_{n}>0\right)$ converges weakly to a probability distribution with g.f. $\mathcal{B}(s)$ satisfying

$$
\mathcal{B}(f(s))-1=m(\mathcal{B}(s)-1), \quad-1<s<1,
$$

that is, $Q(s) \stackrel{\text { def }}{=} \mathcal{B}(s)-1$ is a solution to $(2.3)$. Moreover, $Q^{\prime}(1-)=\mathcal{B}^{\prime}(1-)<\infty$ iff $(L \log L)$ holds true (see [3, Corollary I.11.2]). In view of (3.2) the following lemma is now immediate.

Lemma 3.1. $Q_{n}(s)$ converges pointwise to $Q(s)$ on $(-1,1]$, the convergence being increasing on $[0,1)$ and uniform on every compact subset of $(-1,1)$.

Proof. We only note for the asserted increasing convergence that

$$
\frac{Q_{n+1}(s)}{Q_{n}(s)}=\frac{\left(1-f\left(f_{n}(s)\right)\right) /\left(1-f_{n}(s)\right)}{\left(1-f\left(f_{n}(0)\right)\right) /\left(r 1-f_{n}(0)\right)} \geqslant 1
$$

for all $n \geqslant 0$ and $s \in[0,1)$, where the last inequality holds by the convexity of $f$ on $[0,1)$.

If $(L \log L)$ holds true then, by Theorem I.11.2 in [3], $Q(s)$ must equal a positive constant times

$$
\lim _{n \rightarrow \infty}\left(\left(f_{n}(s)-1\right) / m^{n}\right)
$$

because the latter limit forms another solution to $(2.3)$ with $Q(1)=0$ and finite left derivative $Q^{\prime}(1-)$ at 1 . Without assuming $(L \log L)$ the essential uniqueness of $Q(s)$ as a solution to (2.3) is more difficult. In fact, the following lemma will provide this only within the smaller class of analytic functions on $(-1,1)$ which is fortunately enough for our purposes. A slightly different version of the result is proved in [2, Theorem III.1.2] by different arguments.

Lemma 3.2. The function $Q(s)=\mathcal{B}(s)-1$ forms the unique solution to $(2.3)$ which is analytic on $(-1,1)$ and satisfies $Q(0)=-1$ and $Q(1)=0$.

Proof. Let $R$ be another solution with the desired properties and put $D \stackrel{\text { def }}{=} Q-R$. Then $D(0)=D(1)=0$ and, by iterating (2.3),

$$
D\left(f_{n}(s)\right)=m^{n} D(s)
$$

for all $s \in(-1,1)$. Hence $D\left(f_{n}(0)\right)=0$ for all $n \geqslant 1$. It follows the existence of $\xi \in(0, f(0))$ such that $D^{\prime}(\xi)=0$. We first prove by induction over $k$ that

$$
D^{(k)}\left(f_{n}(\xi)\right)=0
$$

for all $n \geqslant 0$ and $k \geqslant 1$.

Since, by (3.4), $m^{n} D^{\prime}(s)=D^{\prime}\left(f_{n}(s)\right) f_{n}^{\prime}(s)$ for all $n \geqslant 1$ and $s \in(-1,1)$, and since all $f_{n}^{\prime}$ are positive at $\xi$, $D^{\prime}(\xi)=0$ implies (3.5) with $k=1$.

For the inductive conclusion suppose that $D^{(j)}\left(f_{n}(\xi)\right)=0$ for all $n \geqslant 1$ and $1 \leqslant j \leqslant k$. It is easily verified that

$$
m^{n} D^{(j)}(s)=D^{(j)}\left(f_{n}(s)\right)\left(f_{n}^{\prime}\right)^{j}(s)+\sum_{l=1}^{j-1} D^{(l)}\left(f_{n}(s)\right) h_{j, l}(s)
$$

for all $j \geqslant 1$ and suitable functions $h_{j, l}(s)$ which are $\geqslant 0$ for $s \in[0,1)$. Hence the inductive assumption gives

$$
m^{n} D^{(k+1)}\left(f_{i}(\xi)\right)=D^{(k+1)}\left(f_{n+i}(\xi)\right)\left(f_{n}^{\prime}\right)^{k+1}\left(f_{i}(\xi)\right)
$$

for all $i \geqslant 0$ and $n \geqslant 1$. Now, if $D^{(k+1)}\left(f_{i_{0}}(\xi)\right)>0$ then, by (3.6), $D^{(k+1)}\left(f_{n+i_{0}}(\xi)\right)>0$ for all $n \geqslant 0$ in which case we may take ratios on both sides of (3.6) to get

$$
\frac{D^{(k+1)}\left(f_{i}(\xi)\right)}{D^{(k+1)}\left(f_{i+1}(\xi)\right)}=\frac{D^{(k+1)}\left(f_{n+i}(\xi)\right)}{D^{(k+1)}\left(f_{n+i+1}(\xi)\right)} \cdot \frac{\left(f_{n}^{\prime}\right)^{k+1}\left(f_{i}(\xi)\right)}{\left(f_{n}^{\prime}\right)^{k+1}\left(f_{i+1}(\xi)\right)}
$$


for all $i \geqslant 0$ and $n \geqslant 1$. Use $f_{n}^{\prime}\left(f_{i}(\xi)\right)=\prod_{j=0}^{n-1} f^{\prime}\left(f_{j+i}(\xi)\right)$ to infer

$$
\lim _{n \rightarrow \infty} \frac{\left(f_{n}^{\prime}\right)^{k+1}\left(f_{i}(\xi)\right)}{\left(f_{n}^{\prime}\right)^{k+1}\left(f_{i+1}(\xi)\right)}=\lim _{n \rightarrow \infty} \frac{1}{\left(f^{\prime}\right)^{k+1}\left(f_{n+i}(\xi)\right)}=m^{-k-1}
$$

and thus via (3.7) that

$$
\kappa \stackrel{\text { def }}{=} \lim _{n \rightarrow \infty} \frac{D^{(k+1)}\left(f_{n}(\xi)\right)}{D^{(k+1)}\left(f_{n+1}(\xi)\right)}
$$

exists and is positive. However, this is impossible because, by taking the limit $n \rightarrow \infty$ and then $i \rightarrow \infty$ in (3.7), it leads to $\kappa=\kappa m^{-k-1}$ and thus $\kappa=0$. We therefore arrive at the desired conclusion $D^{(k+1)}\left(f_{i}(\xi)\right)=0$ for all $i \geqslant 0$.

To finish the proof of the lemma, we next observe that a Taylor expansion of $D(s)$ about $s=\xi$ in combination with $D^{(k)}(\xi)=0$ for all $k \geqslant 1$ gives that $D(s)=D(\xi)$, i.e. $Q(s)=R(s)+D(\xi)$, for all $s$ in a neighborhood of $\xi$. But then the same must hold true for all $s \in(-1,1)$ because $Q, R$ are analytic. Finally, invoking (2.3) for $Q$ and $R$, we obtain

$$
Q(s)=\frac{Q(f(s))}{m}=\frac{R(f(s))+D(\xi)}{m}=R(s)+\frac{D(\xi)}{m}=Q(s)+\left(\frac{1}{m}-1\right) D(\xi)
$$

and thereby $D(\xi)=0$, i.e. $Q=R$ on $(-1,1)$.

\section{A useful function}

Define the function $\Psi:(0, \infty)^{2} \times((0, \infty) \backslash\{1\}) \rightarrow \mathbb{R}$ by

$$
\Psi(x, y, z) \stackrel{\text { def }}{=} \sum_{n \in \mathbb{Z}}\left(\mathrm{e}^{-x y z^{n}}-\mathrm{e}^{-y z^{n}}\right) .
$$

The connection of $\Psi$ with the $U(\cdot, t)$ in (2.5) is established through the identity

$$
U(s, t)=\Psi\left(-Q(s), m^{-t}, m\right)
$$

for all $s \in[0,1)$ and $t \in \mathbb{R}$, where $Q(0)=-1$ should be recalled. We collect some elementary properties of $\Psi$ in the following lemma.

Lemma 4.1. The function $\Psi$ is well defined and satisfies

$$
\begin{aligned}
& \Psi(x, y, z)=\Psi(x, z y, z) \\
& \Psi(z x, y, z)=\Psi(x, y, z)+\Psi(z, y, z), \\
& \Psi(x, y, z)=\Psi\left(x, y, \frac{1}{z}\right) \\
& \Psi(x, y, z)=\Psi\left(a x, \frac{y}{a}, z\right)+\Psi\left(\frac{1}{a}, y, z\right), \\
& \Psi(x, y, z)=\Psi\left(x, y, z^{2}\right)+\Psi\left(x, z y, z^{2}\right), \\
& \Psi(z, y, z)=\mathbf{1}_{(0,1)}(z)-\mathbf{1}_{(1, \infty)}(z), \\
& \Psi(1, \cdot, z) \equiv 0
\end{aligned}
$$

for all $a, x, y, z>0, z \neq 1$.

Notice that a combination of (4.5) and (4.3) shows that (4.2) generalizes to

$$
U(s, t)=\Psi\left(-Q(s), m^{n-t}, m\right)=\Psi\left(-Q(s), m^{n-t}, 1 / m\right)
$$

for all $s \in[0,1), t \in \mathbb{R}$ and $n \in \mathbb{Z}$.

Proof. (4.3) and (4.5) follow because the definition of $\Psi$ remains unaffected when replacing $n$ with $n+1$, respectively $-n$. Use

$$
\mathrm{e}^{-z x y z^{n}}-\mathrm{e}^{-y z^{n}}=\left(\mathrm{e}^{-x y z^{n+1}}-\mathrm{e}^{-y z^{n+1}}\right)+\left(\mathrm{e}^{-z y z^{n}}-\mathrm{e}^{-y z^{n}}\right)
$$


to get (4.4), and

$$
\mathrm{e}^{-x y z^{n}}-\mathrm{e}^{-y z^{n}}=\left(\mathrm{e}^{-a x \frac{y}{a} z^{n}}-\mathrm{e}^{-\frac{y}{a} z^{n}}\right)+\left(\mathrm{e}^{-\frac{y}{a} z^{n}}-\mathrm{e}^{-y z^{n}}\right), \quad a>0,
$$

to get (4.6). (4.7) follows from

$$
\Psi(x, y, z)=\sum_{n \in \mathbb{Z}}\left(\mathrm{e}^{-x y z^{2 n}}-\mathrm{e}^{-y z^{2 n}}\right)+\sum_{n \in \mathbb{Z}}\left(\mathrm{e}^{-x y z^{2 n+1}}-\mathrm{e}^{-y z^{2 n+1}}\right) .
$$

Since

$$
\Psi(z, y, z)=\lim _{N \rightarrow \infty} \sum_{n=-N}^{N}\left(\mathrm{e}^{-y z^{n+1}}-\mathrm{e}^{-y z^{n}}\right)=\lim _{N \rightarrow \infty}\left(\mathrm{e}^{-y z^{N+1}}-\mathrm{e}^{-y z^{-N}}\right),
$$

we infer (4.8). Finally, (4.9) is obvious from the definition of $\Psi$.

The next three, more difficult lemmata provide us with key tools to identify $\{\eta(t): t \in[0,1)\}$ as the minimal Martin entrance boundary.

Lemma 4.2. For all sufficiently large $z$, the map $[1, z) \ni y \mapsto \Psi(\cdot, y, z)$ is one-to-one.

Proof. For fixed $z>1$, consider $\mathbb{G}(y) \stackrel{\text { def }}{=}\{a>0: \Psi(\cdot, y, z)=\Psi(\cdot, a y, z)\}$ and notice first that $z \in \mathbb{G}(y)$ by (4.3).

Claim 1. $\mathbb{G}(y)=\mathbb{G}(1)$ for all $y>0$.

Fix $y>0$, pick any $a \in \mathbb{G}(y)$, i.e. $\Psi(\cdot, a y, z)=\Psi(\cdot, y, z)$, and use (4.6) to infer

$$
\Psi\left(b x, \frac{y}{b}, z\right)+\Psi\left(\frac{1}{b}, y, z\right)=\Psi\left(b x, \frac{a y}{b}, z\right)+\Psi\left(\frac{1}{b}, a y, z\right)
$$

for all $b, x>0$. Consequently, $\Psi(\cdot, y / b, z)=\Psi(\cdot, a y / b, z)$ and thus $\mathbb{G}(y) \subset \mathbb{G}(y / b)$ for all $b>0$. By symmetry $\mathbb{G}(y)=\mathbb{G}(y / b)$ for all $b>0$ and the claim is proved.

Claim 2. $\mathbb{G} \stackrel{\text { def }}{=} \mathbb{G}(1)$ is a multiplicative group.

Given $a, b \in \mathbb{G}$, we infer $b^{-1} \in \mathbb{G}(a)$ and therefore $\Psi(\cdot, 1, z)=\Psi(\cdot, a, z)=\Psi(\cdot, a / b, z)$ implying $a b^{-1} \in \mathbb{G}$.

Claim 3. $\mathbb{G}$ is closed.

The function $\Psi(x, \cdot, z)$ is continuous for fixed $x, z$. Let $\left(a_{n}\right)_{n} \geqslant 1$ be a sequence in $\mathbb{G}$ which converges to $a>0$. Then $\Psi(x, 1, z)=\lim _{n \rightarrow \infty} \Psi\left(x, a_{n}, z\right)=\Psi(x, a, z)$ for all $x>0$ implies $a \in \mathbb{G}$.

As a closed subgroup of $(0, \infty)$, either $\mathbb{G}=(0, \infty)$ or $\mathbb{G}=\langle a\rangle \stackrel{\text { def }}{=}\left\{a^{n}: n \in \mathbb{Z}\right\}$ for some $a \geqslant 1$. In the second case, $z \in \mathbb{G}$ implies $1<a \leqslant z$ and $a^{n_{0}}=z$ for some $n_{0} \in \mathbb{N}$. Recall that $\mathbb{G}$ depends on the fixed value $z>1$. Now the assertion of the lemma follows if we finally prove

Claim 4. $\mathbb{G}=\langle z\rangle$ for all sufficiently large $z>1$.

Suppose $\mathbb{G} \neq\langle z\rangle$. Then $z^{k / n_{0}} \in \mathbb{G}$ for some $n_{0} \geqslant 2$ and all $k \in \mathbb{Z}$ which implies $\mathbb{G} \cap\left[z^{1 / 3}, z^{2 / 3}\right] \neq \emptyset$ because this intersection contains $y(z) \stackrel{\text { def }}{=} z^{\left\lfloor n_{0} / 2\right\rfloor / n_{0}}$. Since

$$
0=D_{x} \Psi(x, y(z), z)-D_{x} \Psi(x, 1, z)=\sum_{n \in \mathbb{Z}}\left(z^{n} \mathrm{e}^{-x z^{n}}-y(z) z^{n} \mathrm{e}^{-x y(z) z^{n}}\right)
$$

for all $x>0$, where $D_{x}$ denotes the partial derivative w.r.t. $x$, we conclude for all sufficiently large $z$ that 


$$
\begin{aligned}
y(z) \mathrm{e}^{-y(z)}-\mathrm{e}^{-1} & =D_{x} \Psi(1, y(z), z)-D_{x} \Psi(1,1, z)+y(z) \mathrm{e}^{-y(z)}-\mathrm{e}^{-1} \\
& =\sum_{n \geqslant 1}\left(z^{-n} \mathrm{e}^{-z^{-n}}-y(z) z^{-n} \mathrm{e}^{-y(z) z^{-n}}\right)+\sum_{n \geqslant 1}\left(z^{n} \mathrm{e}^{-z^{n}}-y(z) z^{n} \mathrm{e}^{-y(z) z^{n}}\right) \\
& \geqslant-y(z)\left(\sum_{n \geqslant 1} z^{-n}+\sum_{n \geqslant 1} z^{n} \mathrm{e}^{-n z}\right)=-\frac{y(z)}{z-1}-\frac{y(z) z \mathrm{e}^{-z}}{1-z \mathrm{e}^{-z}} .
\end{aligned}
$$

But the first expression of this inequality converges to $-\mathrm{e}^{-1}$, whereas the last one converges to 0 , as $z \rightarrow \infty$. Consequently, the inequality fails to hold for all sufficiently large $z$ and the claim follows.

Lemma 4.3. For all sufficiently large $z$, the set $\mathfrak{C}_{z} \stackrel{\text { def }}{=}\{\Psi(\cdot, y, z): y \in[1, z)\}$ endowed with the metric of pointwise convergence is canonically isomorphic $(y \leftrightarrow \Psi(\cdot, y, z))$ to the compact space $\left([1, z), \rho_{z}\right)$, where

$$
\rho_{z}\left(y_{1}, y_{2}\right) \stackrel{\text { def }}{=} \rho\left(\frac{y_{1}-1}{z-1}, \frac{y_{2}-1}{z-1}\right) .
$$

Furthermore, each $\Psi(\cdot, y, z)$ is minimal in the convex hull of $\mathfrak{C}_{z}$, that is

$$
\Psi\left(\cdot, y_{0}, z\right)=\int_{[1, z)} \Psi(\cdot, y, z) v(\mathrm{~d} y)
$$

for a probability measure $v$ on $[0,1)$ implies $v=\delta_{y_{0}}$.

Plainly, the spaces $\left([1, z), \rho_{z}\right)$ and $([0,1), \rho)$ are isomorphic, too, the first one being an affine linear transformation of the second.

Proof. By the previous lemma, $\mathfrak{C}_{z}$ consists of pairwise distinct elements for sufficiently large $z$. By combining this with the continuity of $\Psi(x, y, z)$ in $y$ and the periodicity property (4.3), the first assertion follows. Now suppose (4.11) for fixed sufficiently large $z$, some $y_{0} \in[1, z)$ and a probability measure $v \neq \delta_{y_{0}}$. We will produce the contradiction that under this assumption $\mathfrak{C}_{z}$ contains no minimal element at all. Using (4.6) for the integrand in (4.11) leads to

$$
\Psi\left(x, y_{0}, z\right)=\int_{[1, z)} \Psi\left(a x, \frac{y}{a}, z\right) v(\mathrm{~d} y)+\int_{[1, z)} \Psi\left(\frac{1}{a}, y, z\right) v(\mathrm{~d} z)
$$

for all $a, x>0$. Setting $x=\frac{1}{a}$, we see with (4.9) that $\Psi\left(\frac{1}{a}, y_{0}, z\right)=\int_{[1, z)} \Psi\left(\frac{1}{a}, y, z\right) v(\mathrm{~d} y)$. By another appeal to (4.6) we hence obtain

$$
\Psi\left(a x, \frac{y_{0}}{a}, z\right)+\Psi\left(\frac{1}{a}, y_{0}, z\right)=\Psi\left(x, y_{0}, z\right)=\int_{[1, z)} \Psi\left(a x, \frac{y}{a}, z\right) v(\mathrm{~d} y)+\Psi\left(\frac{1}{a}, y_{0}, z\right)
$$

for all $a, x>0$ and thus

$$
\Psi\left(x, \frac{y_{0}}{a}, z\right)=\int_{[1, z)} \Psi\left(x, \frac{y}{a}, z\right) v(\mathrm{~d} y)
$$

for all $a, x>0$ which implies the desired contradiction that no $\Psi(\cdot, y, z)$ is minimal.

Lemma 4.4. The assertions of Lemmas 4.2 and 4.3 hold true for all $z>1$.

Proof. It only remains to prove that the map $[1, z) \ni y \mapsto \Psi(\cdot, y, z)$ is one-to-one for all $z>1$. So fix any $z>1$ and suppose $\Psi\left(\cdot, y_{0}, z\right)=\Psi\left(\cdot, y_{1}, z\right)$ for some $1 \leqslant y_{0}, y_{1}<z$. We must show $y_{0}=y_{1}$. Choose $n$ so large that Lemma 4.3 applies to $\mathfrak{C}_{z^{n}}$. A repeated application of (4.7) yields for $i=0,1$

$$
\Psi\left(\cdot, y_{i}, z\right)=\sum_{j=0}^{2^{n}-1} \Psi\left(\cdot, z^{j} y_{i}, z^{2^{n}}\right)=\int \Psi\left(\cdot, y, z^{2^{n}}\right) v_{i, n}(\mathrm{~d} y)
$$


with $v_{i, n} \stackrel{\text { def }}{=} \sum_{j=0}^{2^{n}-1} \delta_{z^{j} y_{i}}$. Hence

$$
\int \Psi\left(\cdot, y, z^{2^{n}}\right) v_{0, n}(\mathrm{~d} y)=\int \Psi\left(\cdot, y, z^{2^{n}}\right) v_{1, n}(\mathrm{~d} y) .
$$

Since all elements of $\mathfrak{C}_{z^{2}}$ are minimal, this equality can only hold if $v_{0, n}=v_{1, n}$ (uniqueness of integral representations) and thus $y_{0}=y_{1}$.

\section{Proof of Theorems 2.1 and 2.2}

Throughout this section the assumptions of Theorem 2.1 as well as the notation of Section 2 are in force. In particular, the definitions of $U(s, t)$ and $\eta(t)$ should be recalled. The proof of Theorem 2.1 is provided by a series of lemmata (5.1)-(5.6) given after the following additional notation and prerequisites.

Recall that $\alpha(x)$ and $\beta(x)$ denote the integral and fractional part of $-\log _{m} x$ for $x>0$. Put $i_{k}^{\prime} \stackrel{\text { def }}{=} i_{k} \zeta_{\tau}\left(i_{k}\right), \alpha_{k} \stackrel{\text { def }}{=} \alpha\left(i_{k}\right)$, $\beta_{k} \stackrel{\text { def }}{=} \beta\left(i_{k}\right), \alpha_{k}^{\prime} \stackrel{\text { def }}{=} \alpha\left(i_{k}^{\prime}\right)$ and $\beta_{k}^{\prime} \stackrel{\text { def }}{=} \beta\left(i_{k}^{\prime}\right)$. Hence $i_{k}=m^{-\alpha_{k}-\beta_{k}}$ and $i_{k}^{\prime}=m^{-\alpha_{k}^{\prime}-\beta_{k}^{\prime}}$. The assumption $\rho\left(i_{k}, t\right) \rightarrow 0$ implies $\beta_{k}^{\prime} \rightarrow t$. Since $i_{k}^{\prime}=i_{k}\left(1-f_{\tau\left(i_{k}\right)}\right) m^{-\tau\left(i_{k}\right)}$ and since $\beta\left(i_{k}\left(1-f_{\tau\left(i_{k}\right)}\right)\right) \in[0,1)$ for large $k$ as argued in Section 2 after the definition of $\tau(x)$, we see that $\alpha_{k}^{\prime}=\tau\left(i_{k}\right)$ and $\beta_{k}^{\prime}=\beta\left(i_{k}\left(1-f_{\tau\left(i_{k}\right)}\right)\right)$ for large $k$.

Defining

$$
c_{N}(k) \stackrel{\text { def }}{=} \inf _{\alpha_{k}^{\prime}-N \leqslant n \leqslant \alpha_{k}^{\prime}+N} \frac{\zeta_{n}}{\zeta_{\alpha_{k}^{\prime}}} \text { and } C_{N}(k) \stackrel{\text { def }}{=} \sup _{\alpha_{k}^{\prime}-N \leqslant n \leqslant \alpha_{k}^{\prime}+N} \frac{\zeta_{n}}{\zeta_{\alpha_{k}^{\prime}}},
$$

and using $\zeta_{1} \geqslant \zeta_{2} \geqslant \cdots$, we infer for any fixed $N \in \mathbb{N}$

$$
\lim _{k \rightarrow \infty} c_{N}(k)=\lim _{k \rightarrow \infty} \frac{\zeta_{\alpha_{k}^{\prime}+N}}{\zeta_{\alpha_{k}^{\prime}}}=\lim _{k \rightarrow \infty} \frac{1-f_{N}\left(f_{\alpha_{k}^{\prime}}(0)\right)}{\left(1-f_{\alpha_{k}^{\prime}}(0)\right) m^{N}}=1,
$$

and similarly

$$
\lim _{k \rightarrow \infty} C_{N}(k)=\lim _{k \rightarrow \infty} \frac{\zeta_{\alpha_{k}^{\prime}-N}}{\zeta_{\alpha_{k}^{\prime}}}=\lim _{k \rightarrow \infty} \frac{\left(1-f_{\alpha_{k}^{\prime}-N}(0)\right) m^{N}}{1-f_{N}\left(f_{\alpha_{k}^{\prime}-N}(0)\right)}=1 .
$$

This will be used in the proof of Lemma 5.3 below.

In order to formulate the first lemma we put

$$
U_{0}(s, t, k, N) \stackrel{\text { def }}{=} \sum_{n=-N}^{N}\left(\exp \left(Q(s) m^{n-\beta_{k}^{\prime}}\right)-\exp \left(-m^{n-\beta_{k}^{\prime}}\right)\right)
$$

for $s, t \in[0,1)$ and $k, N \in \mathbb{N}$. Notice that

$$
U_{0}(s, t, k, N)=\sum_{n=\alpha_{k}^{\prime}-N}^{\alpha_{k}^{\prime}+N}\left(\exp \left(Q(s) i_{k}^{\prime} m^{n}\right)-\exp \left(-i_{k}^{\prime} m^{n}\right)\right) .
$$

Lemma 5.1. If $\rho\left(i_{k}, t\right) \rightarrow 0$, i.e. $\beta_{k}^{\prime} \rightarrow t$, then, for each $s \in[0,1)$ and $\varepsilon>0$, there exists $N_{0} \in \mathbb{N}$ such that

$$
\limsup _{k \rightarrow \infty}\left|U(s, t)-U_{0}(s, t, k, N)\right|<\varepsilon
$$

for all $N \geqslant N_{0}$.

Proof. Fix $s \in[0,1), \varepsilon>0$ and then $N_{0} \in \mathbb{N}$ such that

$$
\left|U(s, t)-\sum_{n=-N}^{N}\left(\exp \left(Q(s) m^{n-t}\right)-\exp \left(-m^{n-t}\right)\right)\right|=\sum_{n:|n|>N}\left(\exp \left(Q(s) m^{n-t}\right)-\exp \left(-m^{n-t}\right)\right)<\varepsilon
$$

for all $N \geqslant N_{0}$. Combining this with 


$$
\begin{aligned}
& \underset{k \rightarrow \infty}{\limsup }\left|U_{0}(s, t, k, N)-\sum_{n=-N}^{N}\left(\exp \left(Q(s) m^{n-t}\right)-\exp \left(-m^{n-t}\right)\right)\right| \\
& \leqslant \sum_{r \in\{0, s\}} \limsup _{k \rightarrow \infty} \sum_{n=-N}^{N}\left|\exp \left(Q(r) m^{n-\beta_{k}^{\prime}}\right)-\exp \left(Q(r) m^{n-t}\right)\right|=0
\end{aligned}
$$

for all $N \in \mathbb{N}$ the assertion easily follows.

Next put $V(s, i) \stackrel{\text { def }}{=} \sum_{j \geqslant 1} G(i, j) s^{j}$ and note that $V(s, i)=\sum_{n \geqslant 0}\left(f_{n}^{i}(s)-f_{n}^{i}(0)\right)$. Put further

$$
V_{0}(s, k, N) \stackrel{\text { def }}{=} \sum_{n=\alpha_{k}^{\prime}-N}^{\alpha_{k}^{\prime}+N}\left(f_{n}^{i_{k}}(s)-f_{n}^{i_{k}}(0)\right)
$$

for $s \in[0,1)$ and $k, N \in \mathbb{N}$. In order to provide a similar result for $V\left(s, i_{k}\right)-V_{0}(s, k, N)$ as in Lemma 5.1, we first prove:

Lemma 5.2. There exists $n_{0} \in \mathbb{N}$ such that

$$
\exp \left(-2 i_{k} \zeta_{n} m^{n}\right) \leqslant f_{n}^{i_{k}}(s) \leqslant \exp \left(-(1-s) i_{k} \zeta_{n} m^{n}\right)
$$

for all $s \in[0,1), k \in \mathbb{N}$ and $n \geqslant n_{0}$.

Proof. Use $1-s=\left|Q_{0}(s)\right| \leqslant\left|Q_{n}(s)\right| \leqslant|Q(s)| \leqslant 1, \log (1+x) \leqslant x$ for $x>-1$, and $\log (1-x) \geqslant-2 x$ for $x \in$ $[0,1 / 2]$ to obtain

$$
f_{n}^{i_{k}}(s)=\left(1+m^{n} \zeta_{n} Q_{n}(s)\right)^{i_{k}}=\exp \left(i_{k} \log \left(1+m^{n} \zeta_{n} Q_{n}(s)\right)\right) \leqslant \exp \left(-(1-s) i_{k} \zeta_{n} m^{n}\right)
$$

for $s \in[0,1)$ and $n \geqslant 0$, and similarly the left inequality in (5.3) for $s \in[0,1)$ and $n \geqslant n_{0}$ for a suitably chosen $n_{0} \in \mathbb{N}$ not depending on $s$ or $k$.

Lemma 5.3. For each $s \in[0,1)$ and $\varepsilon>0$, there exists $N_{0} \in \mathbb{N}$ such that

$$
\limsup _{k \rightarrow \infty}\left|V\left(s, i_{k}\right)-V_{0}(s, k, N)\right|<\varepsilon
$$

for all $N \geqslant N_{0}$.

Proof. Let $k$ be so large that $\alpha_{k}^{\prime}=\tau\left(i_{k}\right)$, hence $i_{k}^{\prime}=i_{k} \zeta_{\alpha_{k}^{\prime}}$, and consider

$$
\begin{aligned}
& V_{1}(s, k, N) \stackrel{\text { def }}{=} \sum_{n=0}^{\alpha_{k}^{\prime}-N-1}\left(f_{n}^{i_{k}}(s)-f_{n}^{i_{k}}(0)\right), \\
& V_{2}(s, k, N) \stackrel{\text { def }}{=} \sum_{n>\alpha_{k}^{\prime}+N}\left(f_{n}^{i_{k}}(s)-f_{n}^{i_{k}}(0)\right) .
\end{aligned}
$$

The assertion obviously follows if we prove that the terms $V_{1}$ and $V_{2}$ become small for $k, N$ sufficiently large. Let $l \in \mathbb{N}$ be such that $f_{l-1}(0)<s \leqslant f_{l}(0)$. Then

$$
V_{1}(s, k, N) \leqslant \sum_{n=0}^{\alpha_{k}^{\prime}-N-1}\left(f_{n+l}^{i_{k}}(0)-f_{n}^{i_{k}}(0)\right)=\sum_{n=\alpha_{k}^{\prime}-N}^{\alpha_{k}^{\prime}-N+l-1} f_{n}^{i_{k}}(0)-\sum_{n=0}^{l-1} f_{n}^{i_{k}}(0) .
$$

The second sum in the previous line clearly converges to 0 as $k \rightarrow \infty$. As to the first sum, we infer with Lemma 5.2 


$$
\begin{aligned}
\sum_{n=\alpha_{k}^{\prime}-N}^{\alpha_{k}^{\prime}-N+l-1} f_{n}^{i_{k}}(0) & \leqslant \sum_{n=\alpha_{k}^{\prime}-N}^{\alpha_{k}^{\prime}-N+l-1} \exp \left(-i_{k} m^{n} \zeta_{n}\right) \leqslant l \exp \left(-i_{k} \zeta_{\alpha_{k}^{\prime}} c_{N}(k) m^{\alpha_{k}^{\prime}-N+l-1}\right) \\
& =l \exp \left(-c_{N}(k) m^{-N+l-\beta_{k}^{\prime}-1}\right) \leqslant l \exp \left(-c_{N}(k) m^{-N+l-1}\right)
\end{aligned}
$$

and therefore with (5.1)

$$
\limsup _{k \rightarrow \infty} \sum_{n=\alpha_{k}^{\prime}-N}^{\alpha_{k}^{\prime}-N+l-1} f_{n}^{i_{k}}(0) \leqslant l \exp \left(-m^{-N+l-1}\right)
$$

which can be made arbitrarily small if $N$ is chosen large enough.

As to $V_{2}$, we obtain by another appeal to Lemma 5.2

$$
\begin{aligned}
V_{2}(s, k, N) & \leqslant \sum_{n>\alpha_{k}^{\prime}+N}\left(f_{n+l}^{i_{k}}(0)-f_{n}^{i_{k}}(0)\right)=\sum_{n=\alpha_{k}^{\prime}+N+1}^{\alpha_{k}^{\prime}+N+l}\left(1-f_{n}^{i_{k}}(0)\right) \leqslant \sum_{n=\alpha_{k}^{\prime}+N+1}^{\alpha_{k}^{\prime}+N+l} \exp \left(-2 i_{k} \zeta_{n} m^{n}\right) \\
& \leqslant l\left(1-\exp \left(-2 i_{k} \zeta_{\alpha_{k}^{\prime}} c_{N+l}(k) m^{\alpha_{k}^{\prime}+N+1}\right)\right) \leqslant l\left(1-\exp \left(-2 c_{N+l}(k) m^{N+1}\right)\right)
\end{aligned}
$$

so that upon using (5.2)

$$
\limsup _{k \rightarrow \infty} V_{2}(s, k, N) \leqslant l\left(1-\exp \left(-2 m^{N+1}\right)\right)
$$

which again becomes arbitrarily small if $N$ is large enough.

A major step towards the proof of Theorem 2.1 is provided by the next lemma

Lemma 5.4. For any integer sequence $\left(i_{k}\right)_{k} \geqslant 1, \rho\left(i_{k}, t\right) \rightarrow 0$ for some $t \in[0,1)$ implies that $G\left(i_{k}, \cdot\right)$ converges pointwise to the quasi-invariant Radon measure $\eta(t)$.

Proof. It suffices to show that $\lim _{k \rightarrow \infty} V\left(s, i_{k}\right)=U(s, t)$ for $s \in[0,1)$. This was also done in [3, pp. 70 and 100f] under $(L \log L)$, but an argument at the end of their proof does not work under the $(L \log L)$-condition (see the remark after this lemma for further details). A refinement of their argument is therefore needed even if $(L \log L)$ holds true.

Fix $s \in[0,1)$, an arbitrary $\varepsilon>0$ and then $N$ so large that, by Lemmata 5.1 and 5.3,

$$
\limsup _{k \rightarrow \infty}\left|U(s, t)-U_{0}(s, t, k, N)\right|<\frac{\varepsilon}{2} \text { and } \limsup _{k \rightarrow \infty}\left|V\left(s, i_{k}\right)-V_{0}(s, k, N)\right|<\frac{\varepsilon}{2} .
$$

Hence

$$
\limsup _{k \rightarrow \infty}\left|V\left(s, i_{k}\right)-U(s, t)\right| \leqslant \limsup _{k \rightarrow \infty}\left|V_{0}(s, k, N)-U_{0}(s, t, k, N)\right|+\varepsilon
$$

which leaves us with the proof of

$$
\lim _{k \rightarrow \infty}\left|V_{0}(s, k, N)-U_{0}(s, t, k, N)\right|=0 .
$$

Put

$$
D(s, k, N) \stackrel{\text { def }}{=} \sum_{n=\alpha_{k}^{\prime}-N}^{\alpha_{k}^{\prime}+N}\left(f_{n}^{i_{k}}(s)-\exp \left(i_{k}^{\prime} Q(s) m^{n}\right)\right)
$$

and note that $V_{0}(s, k, N)-U_{0}(s, t, k, N)=D(s, k, N)-D(0, k, N)$. Let $k$ be so large that $\alpha_{k}^{\prime}=\tau\left(i_{k}\right)$ and thus $i_{k}^{\prime}=i_{k} \zeta_{\alpha_{k}^{\prime}}$. Using $\left|x^{k}-y^{k}\right| \leqslant k|x-y|$ for $x, y \in[0,1]$ we obtain

$$
|D(s, k, N)| \leqslant i_{k} \sum_{n=\alpha_{k}^{\prime}-N}^{\alpha_{k}^{\prime}+N}\left|f_{n}(s)-\exp \left(\zeta_{\alpha_{k}^{\prime}} Q(s) m^{n}\right)\right|
$$


for $s \in[0,1)$. Since $\left|e^{x}-1-x\right| \leqslant x^{2}$ for $|x| \leqslant 1$, we further see with $i_{k}^{\prime}=m^{-\alpha_{k}^{\prime}-\beta_{k}^{\prime}}$ that

$$
\begin{aligned}
i_{k} \sum_{n=\alpha_{k}^{\prime}-N}^{\alpha_{k}^{\prime}+N}\left|\exp \left(\zeta_{\alpha_{k}^{\prime}} Q(s) m^{n}\right)-1-\zeta_{\alpha_{k}^{\prime}} Q(s) m^{n}\right| & \leqslant i_{k}^{\prime} \sum_{n=\alpha_{k}^{\prime}-N}^{\alpha_{k}^{\prime}+N} Q(s)^{2} m^{2 n} \leqslant i_{k}^{\prime} m^{2\left(\alpha_{k}^{\prime}-N\right)} /\left(1-m^{2}\right) \\
& =m^{\alpha_{k}^{\prime}-2 N-\beta_{k}^{\prime}} /\left(1-m^{2}\right) \leqslant m^{\alpha_{k}^{\prime}-2 N-1} /\left(1-m^{2}\right),
\end{aligned}
$$

which converges to 0 as $k \rightarrow \infty$. Combining this with $f_{n}(s)-1=\zeta_{n} Q_{n}(s) m^{n}$ for $n \geqslant 0$ and setting

$$
\Lambda_{N}(k) \stackrel{\text { def }}{=}\left|c_{N}(k)-1\right| \vee\left|C_{N}(k)-1\right|,
$$

we infer in (5.5)

$$
\begin{aligned}
|D(s, k, N)| & \leqslant \mathrm{o}(1)+i_{k} \sum_{n=\alpha_{k}^{\prime}-N}^{\alpha_{k}^{\prime}+N}\left|f_{n}(s)-1-\zeta_{\alpha_{k}^{\prime}} Q(s) m^{n}\right| \\
& \leqslant \mathrm{o}(1)+i_{k} \sum_{n=\alpha_{k}^{\prime}-N}^{\alpha_{k}^{\prime}+N} m^{n}\left(\left|\zeta_{n} Q_{n}(s)-\zeta_{\alpha_{k}^{\prime}} Q_{\alpha_{k}^{\prime}}(s)\right|+\zeta_{\alpha_{k}^{\prime}}\left|Q_{\alpha_{k}^{\prime}}(s)-Q(s)\right|\right) \\
& \leqslant \mathrm{o}(1)+i_{k}^{\prime} \sum_{n=\alpha_{k}^{\prime}-N}^{\alpha_{k}^{\prime}+N} m^{n}\left(\left|Q_{\alpha_{k}^{\prime}}(s)\right| \Lambda_{N}(k)+\left|Q_{\alpha_{k}^{\prime}}(s)-Q(s)\right|\right) \\
& \leqslant \mathrm{o}(1)+i_{k}^{\prime} m^{\alpha_{k}^{\prime}-N}(1-m)^{-1}\left(\Lambda_{N}(k)+\left|Q_{\alpha_{k}^{\prime}}(s)-Q(s)\right|\right) \\
& \leqslant \mathrm{o}(1)+m^{-N-1}(1-m)^{-1}\left(\Lambda_{N}(k)+\left|Q_{\alpha_{k}^{\prime}}(s)-Q(s)\right|\right)
\end{aligned}
$$

as $k \rightarrow \infty$. Since $\Lambda_{N}(k) \rightarrow 0$ by (5.1), (5.2) and $Q_{\alpha_{k}^{\prime}}(s) \rightarrow Q(s)$ by Lemma 3.1, we have proved

$$
\lim _{k \rightarrow \infty} D(s, k, N)=0
$$

for all $s \in[0,1)$ and therefore also (5.4).

Remark. By a simpler estimation than ours, Athreya and Ney [3, p. 100f] showed that $\lim _{k \rightarrow \infty} V\left(s, i_{k}\right)=U(s, t)$ follows from

$$
\sum_{n \geqslant 1}\left|Q_{n}(s)-Q(s)\right|<\infty
$$

and claim this in turn be true under $(L \log L)$. However, assertion (5.6) actually requires the stronger moment assumption $\left(L \log ^{2} L\right)$, i.e. $\sum_{j \geqslant 1} p_{j} j \log ^{2} j<\infty$, and is therefore too weak to give Lemma 5.4 under $(L \log L)$.

The next lemma is the converse of the previous one.

Lemma 5.5. If $G\left(i_{k}, \cdot\right)$ converges pointwise to a quasi-invariant Radon measure $\eta$ then $\rho\left(i_{k}, t\right) \rightarrow 0$ for some $t \in[0,1)$ and $\eta=\eta(t)$.

Proof. Since $(\mathbb{M}, \rho)$ is a compact space, $\left(i_{k}\right)_{k} \geqslant 1$ contains a $\rho$-convergent subsequence $\left(j_{k}\right)_{k} \geqslant 1$. If $j_{k}$ were eventually constant, i.e. $j_{k_{0}}=j_{k_{0}+1}=\cdots=j$ for some $k_{0}, j \geqslant 1$, then $\eta=\lim _{k \rightarrow \infty} G\left(j_{k}, \cdot\right)=G(j, \cdot)$ which is impossible because $G(j, \cdot)$ is not quasi-invariant. Consequently, $j_{k} \rightarrow \infty$ and $\rho\left(j_{k}, t\right) \rightarrow 0$ for some $t \in[0,1)$. Now we conclude from the first part of the lemma that $\eta=\lim _{k \rightarrow \infty} G\left(j_{k}, \cdot\right)=\eta(t)$.

Lemma 5.6. The $\eta(t), t \in[0,1)$, are pairwise distinct and minimal. 
Proof. We consider the g.f. $U(\cdot, t)$ of $\eta(t)$ and recall from (4.10) that $U(s, t)=\Psi\left(-Q(s), m^{n-t}, m\right)$ for all $(s, t) \in$ $[0,1)^{2}$ and $n \in \mathbb{Z}$. Suppose that $U\left(\cdot, t_{1}\right)=U\left(\cdot, t_{2}\right)$ for $t_{1}, t_{2} \in[0,1)$. Since $Q(s)$ is strictly increasing from -1 to 0 for $s \in[0,1)$, we infer $\Psi\left(\cdot, m^{-t_{1}}, m\right)=\Psi\left(\cdot, m^{-t_{2}}, m\right)$ on $(0,1]$. But both functions are also easily seen to be complex differentiable in the open complex strip $(0, \infty) \times \mathrm{i} \mathbb{R}$. Since holomorphic functions are uniquely determined by their values on an interval, we conclude $\Psi\left(\cdot, m^{-t_{1}}, m\right)=\Psi\left(\cdot, m^{-t_{2}}, m\right)$ on the whole strip $(0, \infty) \times \mathrm{i} \mathbb{R}$. Hence Lemmata 4.3 and 4.4 ensure that $m^{-t_{1}}=m^{-t_{2}}$ and thus $t_{1}=t_{2}$. This proves the pairwise distinctness of the $\eta(t)$.

Minimality follows by a similar argument. If

$$
U\left(\cdot, t_{0}\right)=\int_{[0,1)} U(\cdot, t) v(\mathrm{~d} t)
$$

for $t_{0} \in[0,1)$ and a finite measure $v$ on $[0,1)$, then we infer

$$
\Psi\left(\cdot, m^{-t_{0}}, m\right)=\int_{[0,1)} \Psi\left(\cdot, m^{-t}, m\right) v(\mathrm{~d} t)
$$

on the whole strip $(0, \infty) \times \mathrm{i} \mathbb{R}$ and thus $v=\delta_{t_{0}}$ by another appeal to Lemmata $4.3,4.4$.

Proof of Theorem 2.2. It obviously suffices to show that $\rho\left(i_{k}, t\right) \rightarrow 0$ and $\rho^{*}\left(i_{k}, t\right) \rightarrow 0$ are equivalent for $t \in[0,1)$ and any integer sequence $\left(i_{k}\right)_{k} \geqslant 1$. Write $a \equiv b\left[m^{\mathbb{Z}}\right]$ to mean that $a / b=m^{n}$ for some $n \in \mathbb{Z}$. We have

$$
i_{k} \zeta_{\tau\left(i_{k}\right)} \equiv m^{\beta_{k}^{\prime}}\left[m^{\mathbb{Z}}\right] \quad \text { and } i_{k} \zeta \equiv m^{\beta_{k}^{\prime \prime}}\left[m^{\mathbb{Z}}\right]
$$

for suitable $\beta_{k}^{\prime}, \beta_{k}^{\prime \prime} \in[0,1)$ and thus, by taking the ratio,

$$
\frac{\zeta_{\tau\left(i_{k}\right)}}{\zeta} \equiv m^{\beta_{k}^{\prime}-\beta_{k}^{\prime \prime}}\left[m^{\mathbb{Z}}\right]
$$

Since, given $(L \log L)$, the left-hand side converges to 1 as $k \rightarrow \infty$ and since $\beta_{k}^{\prime}-\beta_{k}^{\prime \prime} \in(-1,1)$ for all $k \geqslant 1$, we conclude that $\beta_{k}^{\prime} \rightarrow t$, i.e. $\rho\left(i_{k}, t\right) \rightarrow 0$, holds iff $\beta_{k}^{\prime \prime} \rightarrow t$, i.e. $\rho^{*}\left(i_{k}, t\right) \rightarrow 0$.

\section{References}

[1] G. Alsmeyer, U. Rösler, Asexual versus promiscuous bisexual Galton-Watson processes: The extinction probability ratio, Ann. Appl. Probab. 12 (2002) 125-142.

[2] S. Asmussen, H. Hering, Branching Processes, Birkhäuser, Boston, 1983.

[3] K.B. Athreya, P. Ney, Branching Processes, Springer, New York, 1972.

[4] T.E. Harris, The Theory of Branching Processes, Springer, Heidelberg, 1963.

[5] P. Jagers, Branching Processes with Biological Applications, Wiley, London, 1975.

[6] S. Karlin, J. McGregor, Uniqueness of stationary measures for branching processes and applications, in: Proc. of the Fifth Berkeley Symposium, vol. II, Univ. of California Press, Berkeley, 1967, pp. 243-254.

[7] J.G. Kemeny, J.L. Snell, A.W. Knapp, Denumerable Markov Chains, second ed., Springer, New York, 1976.

[8] H. Kesten, P. Ney, F. Spitzer, The Galton-Watson process with mean one and finite variance, Theory Probab. Appl. 11 (1966) 513-540.

[9] J.F.C. Kingman, Stationary for branching processes, Proc. Amer. Math. Soc. 16 (1965) 245-247.

[10] F. Papangelou, A lemma on the Galton-Watson process and some of its consequences, Proc. Amer. Math. Soc. 19 (1968) 1469-1479.

[11] E. Seneta, The Galton-Watson process with mean one, J. Appl. Probab. 4 (1967) 489-495.

[12] F. Spitzer, Two explicit Martin boundary constructions, in: Symposium on Probab. Methods in Analysis, in: Lecture Notes in Math., vol. 31 , Springer, Berlin, 1967, pp. 296-298. 